Review

\title{
Calibration procedure for 3D measurement systems using two cameras and a laser line
}

\author{
João L. Vilaça ${ }^{\mathrm{a}, *}$, Jaime C. Fonseca ${ }^{\mathrm{a}}$, António M. Pinho ${ }^{\mathrm{b}}$ \\ a Industrial Electronic Department, University of Minho, Campus Azurem, 4800-058 Guimarães, Portugal \\ ${ }^{\mathrm{b}}$ Mechanical Engineering Department, Minho University, Campus Azurem, 4800-058 Guimarães, Portugal
}

\section{A R T I C L E I N F O}

\section{Article history:}

Received 19 November 2007

Received in revised form

12 May 2008

Accepted 13 May 2008

\section{Keywords:}

Calibration

Laser line detection

3D measurements

\begin{abstract}
A B S T R A C T
In order to ensure the precision of the measurement of complex 3D object surfaces using non-contact laser scanning systems, a novel stereo vision calibration procedure based on a laser line projection plane is presented. This calibration procedure can also be used in measurement systems based on a single camera and a laser line projection. This procedure, while using only laser-coplanar points, is oriented towards laser line detection and allows the matching of two images on the laser projection plane without the use of a rigid motion equation. These features make this procedure very precise, simple and, consequently, easier to implement.
\end{abstract}

(c) 2008 Elsevier Ltd. All rights reserved.

\section{Contents}

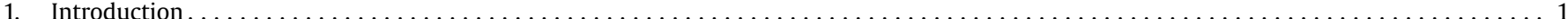

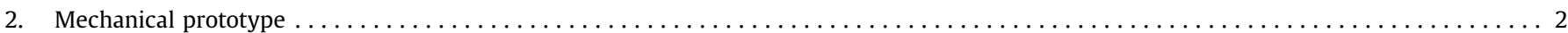

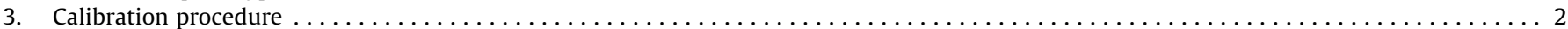

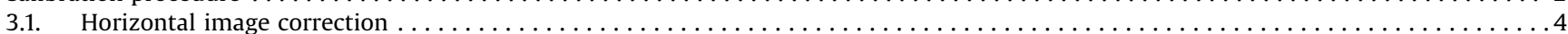

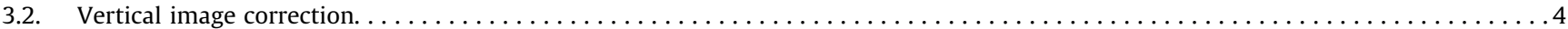

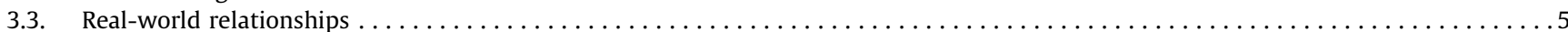

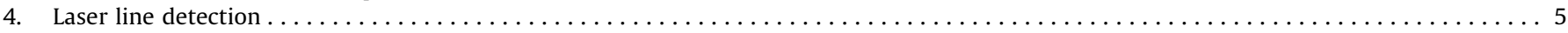

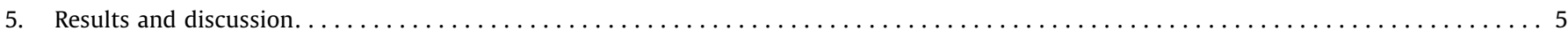

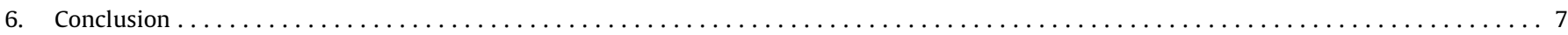

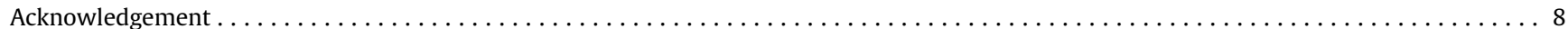

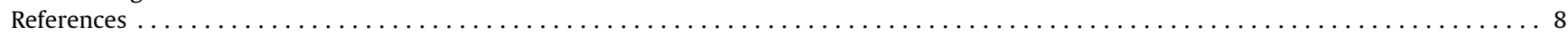

\section{Introduction}

Nowadays, reverse engineering from physical models has two principal and distinct goals: the comparison between the real dimensions of a sculptured model and its theoretical dimensions and the generation of computer-based models (CAD) from a physical model with complex geometry [1].

Some of these measurement techniques are based on scanning a surface, being characterized by a direct controlled energy projection onto the surface, resorting to one or more projectors

\footnotetext{
* Corresponding author. Tel.: +351 253510190; fax: +351253510189.

E-mail address: joaovilaca@dei.uminho.pt (J.L. Vilaça).
}

and one or more sensors. These techniques require complicated calibration procedures to evaluate the parameters of the optical devices (sensors and projectors) necessary for the measurement process [2].

Camera (sensor) calibration is understood as the process of determining the internal camera geometric and optical characteristics (intrinsic parameters) and/or the 3D position and orientation of the camera frame relative to a certain world coordinate system (extrinsic parameters). The purpose of the calibration is to establish a relationship between 3D points from the world and 2D points from the image seen by the computer [3-7].

In 3D measurements systems, composed of one laser light and one camera, the common procedure is to use the triangulation method (active vision technique) to establish the relationship

0030-3992/\$- see front matter ( 2008 Elsevier Ltd. All rights reserved. doi:10.1016/j.optlastec.2008.05.012 


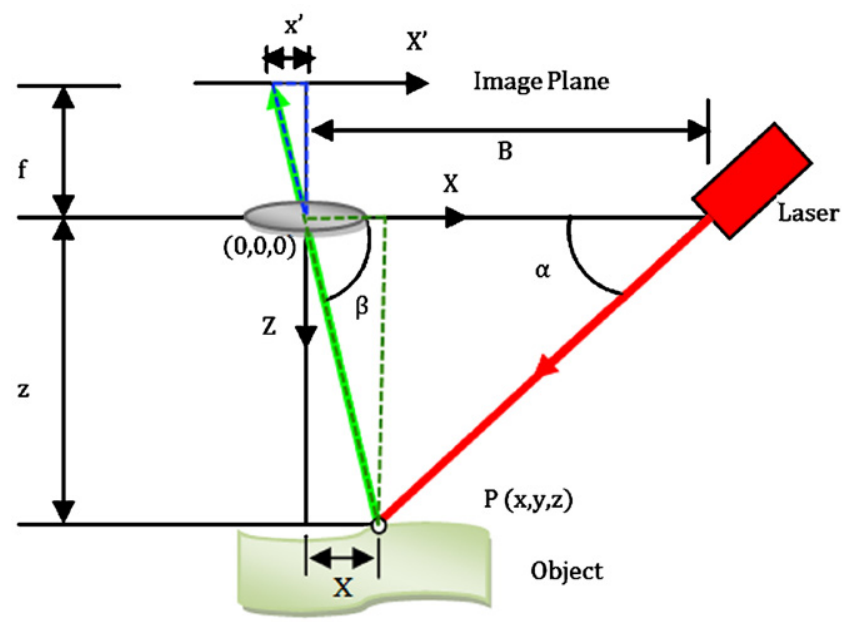

Fig. 1. Laser triangulation geometry.

between $2 \mathrm{D}$ points from the image and the $3 \mathrm{D}$ points from the world [8]. Fig. 1(a) illustrates the basic principle of laser triangulation. In this technique, the distance to the object $z$ is calculated using trigonometrical relationships, based on the relative positions and orientations $(\alpha$ and $\beta$ ) of the camera and laser, by setting the origin of the axes on the centre of the lenses, given that the focal distance $f$ (distance between the lens and the image plane) and the distance between the laser and the camera (base line B) are known. So, rather than measuring the angles directly $(\alpha$ and $\beta$ ), the laser triangulation is based on triangle similarity: comparing the object triangle (coloured green in Fig. 1(b)) and the image triangle (coloured blue in Fig. 1(b)).

One of the major limitations of this technique is the occlusion problem, which occurs when the camera is not capable of visualizing the laser beam reflected by the object, due to the object geometry. To overcome this limitation, it is common to use a second camera. However, the use of two cameras involves a stereo match between the points of the right image and the points of the left image, associated with the same physical point of the scene 3D-the stereo matching problem. The matching process is typically based on similarity constrains (feature based or intensity based). Nevertheless, in epipolar geometry, a point on the right image can be associated with many points on the left image (false matches). This problem is particularly relevant in the measurement of free-form surfaces due to the absence of particularities in the scene, used as similarity points [9]. Some calibration procedures can solve the matching problem by intersecting the epipolar line with the laser line in the image [10].

This paper presents a new stereo vision calibration procedure based on a laser line projection (plane) for non-contact laser scanning systems. The calibration procedure, while using only laser-coplanar points, is oriented towards laser line detection, allowing the matching of two images on the laser plane without the use of a rigid motion equation and correcting two kinds of distortion, radial distortion and distortion provoked by the orientation of the camera relative to the laser line projection plane.

The paper is divided into the following sections: Section 2 describes the mechanical prototype developed and the configuration of the scanning arm; Section 3 explains the calibration procedure developed and is composed of three sub-sections dealing with: horizontal image correction, vertical image correction and real-world relationship; Section 4 presents an algorithm for laser line detection on surfaces with high laser line absorption; and finally Section 5 presents practical applications of the system developed on different kinds of surfaces.

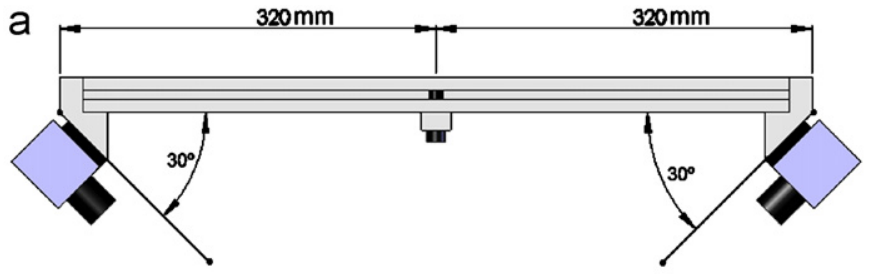

b

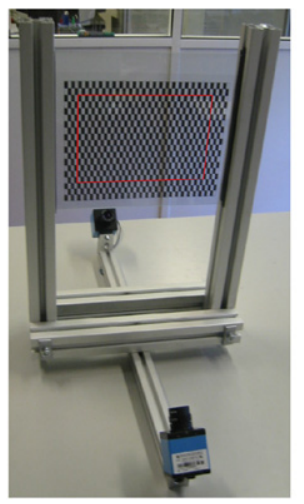

Fig. 2. Scanning arm: (a) dimensions and geometrical relations, (b) calibration setup (scanner arm assembled in the pattern support).

\section{Mechanical prototype}

The mechanical prototype built is a 3D freedom machine with a working area of $1000 \times 1000 \times 500 \mathrm{~mm}^{3}$ and is controlled by a PC-based motion control system.

The scanning arm is composed of two CCD FIREWIRE cameras, with a resolution of $640 \times 480$, using $8 \mathrm{~mm}$ focal length lenses and a $635 \mathrm{~nm}$ wavelength, $1 \mathrm{~mW}$ power laser line. The laser is located at the same physical distance from both cameras, and the cameras are oriented at an angle of $30^{\circ}$ to the horizontal line (Fig. 2(a))

\section{Calibration procedure}

Most of the procedures used for camera calibration are normally based on the pinhole camera model. In the first step, the camera image is transformed into an ideal (undistorted) image, using perspective projection with pinhole camera geometry, thus taking into account the intrinsic camera parameters and modelling lens distortion, which can be of two kinds: radial and tangential. Then, the calibration parameters $R$ (rotation matrix) and $T$ (translation vector) are defined, allowing, through a rigid equation, the transformation of the camera coordinate system in the object world coordinate system.

In the proposed calibration procedure, only radial distortion is considered because it is the most relevant distortion to industrial machine vision applications, and only the laser line projection plane is calibrated because measurements are only made along the plane defined by the laser line.

The laser is located at the same physical distance from both cameras, and the cameras are oriented at $30^{\circ}$ to the horizontal line. Although, the system is mechanically adjusted (distance between cameras and laser, angles between cameras and laser line), the acquired images of both cameras showed that, in the laser line projection plane, the system was far from being calibrated, presenting the following problems:

- A point $P($ real) in the Olaser (laser coordinates system), corresponds to a point $P 1$ in the 01 (camera 1 coordinates 
system), and another point $P 2$ in the 02 (camera 2 coordinates system), being $P 1$ different from $P 2$.

- Perfectly visible radial distortion in some parts of the image, adding to the previous problem.

- Formation of image dead zones-problem caused by the relative angle of the cameras to the laser plane. This distortion means that the horizontal length of the vision field at the top of the image is smaller than the horizontal length of the vision

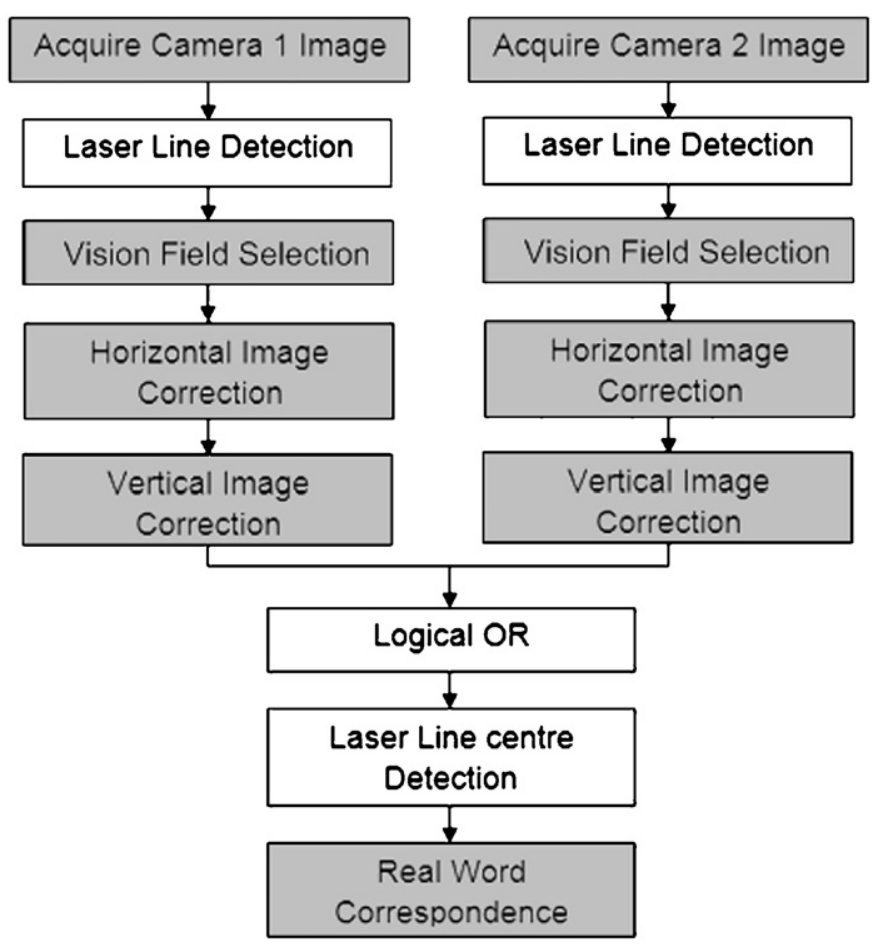

Fig. 3. Flowchart of the proposed calibration procedure including laser line detection. field at the bottom of the image. This problem only matters because the system to be calibrated is assembled in a 3D freedom machine that will carry out readings on parallel surface planes.

In order to overcome these problems, a calibration procedure was developed which calibrates each image, asserting that a point $P 1^{\prime}$ in $O_{1}$ is equal to a point $P 2^{\prime}$ in $O_{2}$ on the laser plane, eliminating the radial distortion caused by the lenses and image dead zones. The flowchart of the proposed calibration procedure including laser line detection is presented in Fig. 3 .

For calibration, a pattern was created with $10 \times 5 \mathrm{~mm}^{2}$ black and white rectangles. The pattern was printed on a transparency sheet and placed between two glasses with a thickness of $1.2 \mathrm{~mm}$, all the rectangles being co-planar and visible to both the cameras (Fig. 2(b)). The pattern must then be aligned along the laser line projection plane allowing an easy match between the same image points seen by both cameras, which is the basis of this calibration procedure (Fig. 4).

The calibration procedure starts with the capture of a pattern image by each camera.

After both images have been acquired, a valid vision field is defined for each camera (Fig. 5(a)). The selected vision field must be the same for both cameras; the horizontal and vertical limits of the selected vision field must correspond to the same physical points of the pattern.

The third step of the calibration procedure consists in the horizontal calibration of the acquired images. In this step, the horizontal correction of the images is made (Fig. 5(b)), resulting in two new horizontally undistorted images (Fig. 5(c)).

The fourth step concerns the vertical calibration of the resultant images of the previous step. These two new images are horizontally and vertically distortions free (Fig. 5(d)).

At the end of above four steps, pixels with same coordinates in images acquired from each camera correspond to the same physical location of the pattern.

As a result, data obtained from an image acquired by camera 1 can now be used to complement data extracted from the

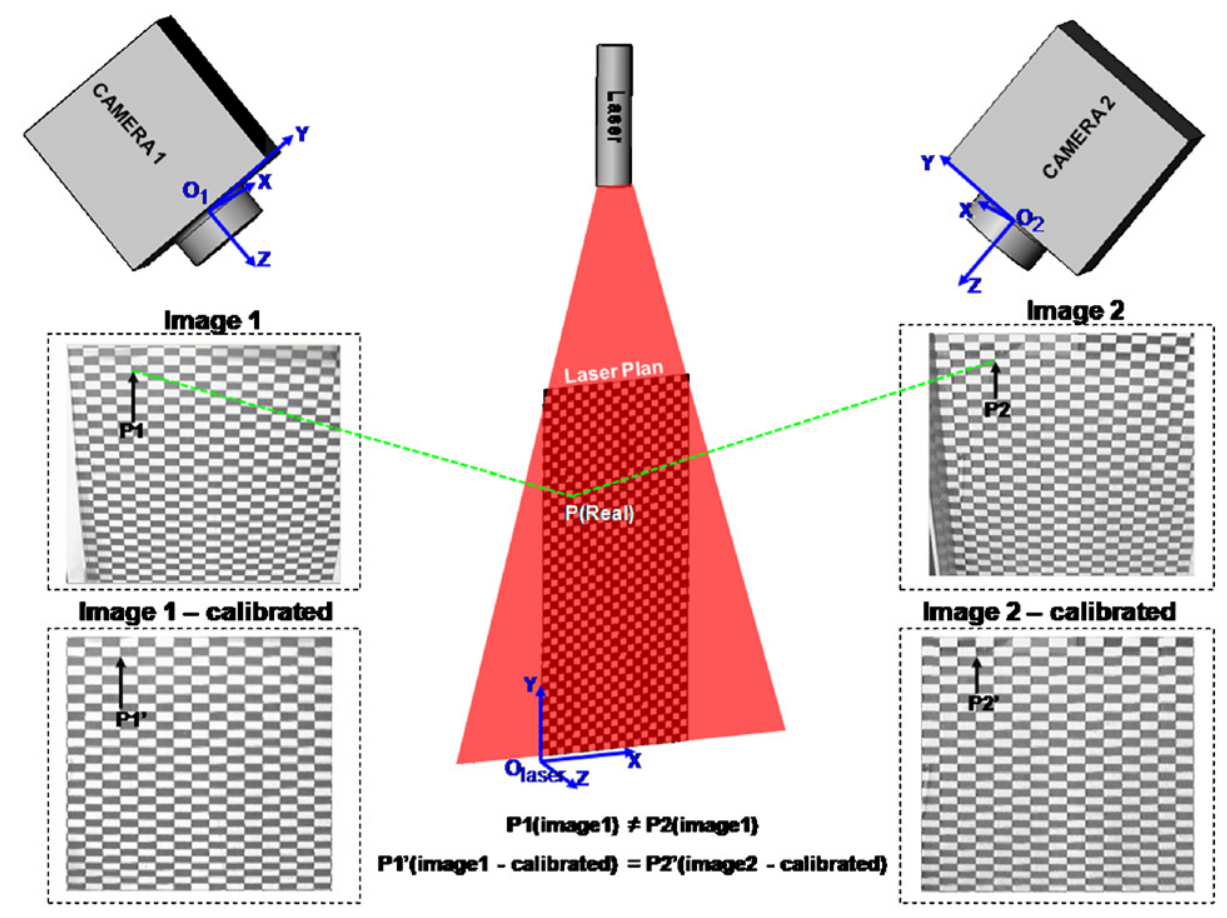

Fig. 4. Calibration procedure illustration and correspondence of one point in $O_{\text {laser }}$ plane in the two camera images, before and after calibration. 
a

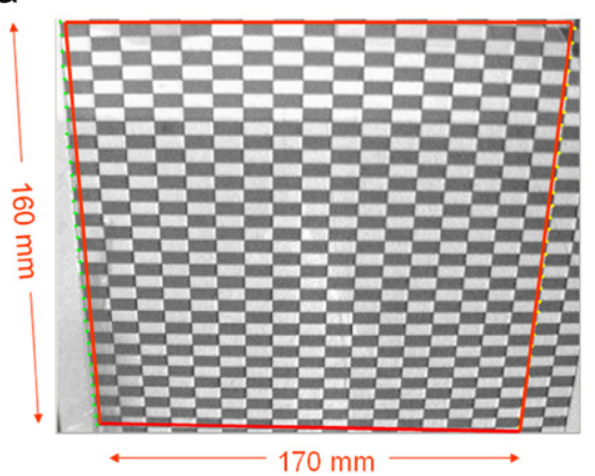

C

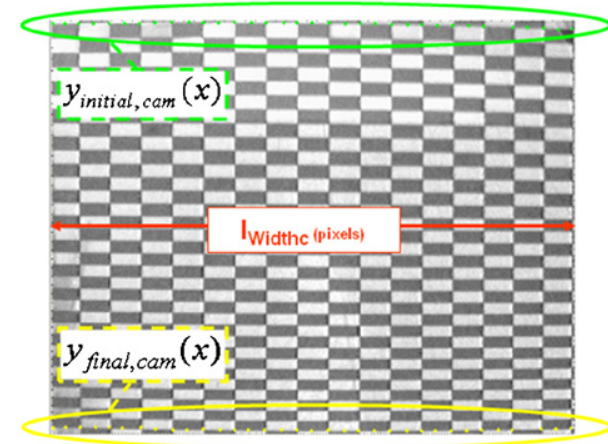

b

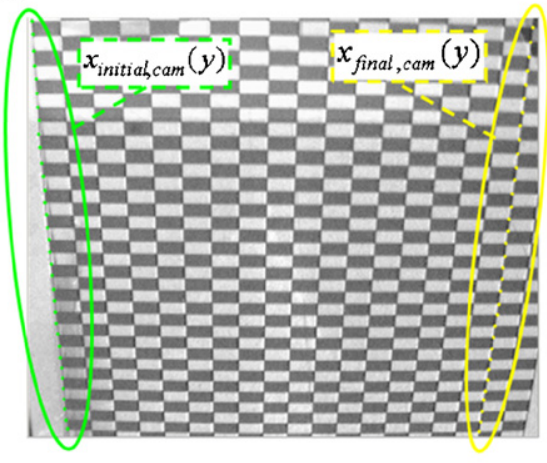

d

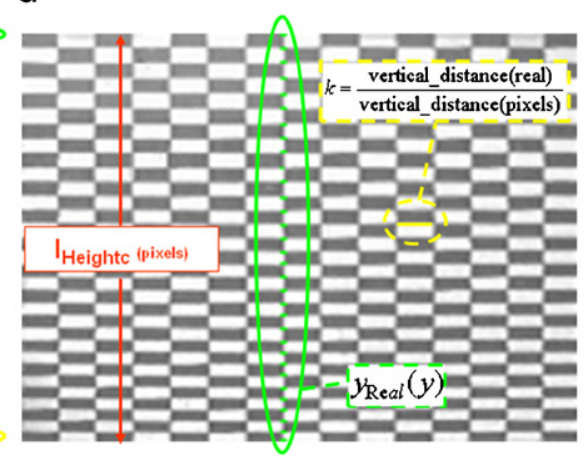

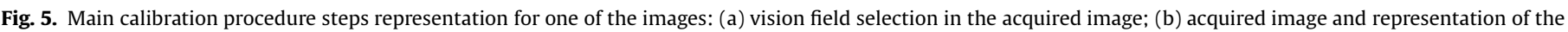

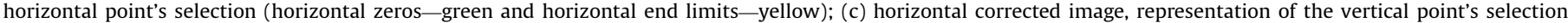

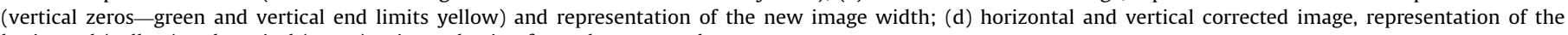
horizontal (yellow) and vertical (green) points selection for real correspondence.

corresponding image acquired by camera 2, particularly in the case of object occlusion problem.

In the last step of the calibration procedure it is necessary to set a relationship between the image points and the pattern points (Fig. 5(d)) -a linear relationship in the horizontal component and a polynomial relationship in the vertical component. The determination of those relationships needs to be applied to one image only.

The horizontal image correction, vertical image correction and real-world relationships are described in detail in the following sections.

The calibration procedure also includes the automatic laser line detection as will be described in Section 4 .

\subsection{Horizontal image correction}

The horizontal image correction (Fig. 5(b)) for each camera is divided into the following steps (the points acquired from each image will have to correspond to the horizontal limits of the selected vision field):

(a) Acquisition of a set of vertical points from the image, which will be the horizontal zeros of the image after horizontal image correction.

(b) Polynomial determination that translates the relation between the position $(y)$ of vertical points and the position $(x)$ of

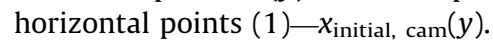

(c) Acquisition of a set of vertical points from the image, which will be the horizontal end limits of the image after horizontal image correction.

(d) Polynomial determination that translates the relation between the position $(y)$ of vertical points and the position $(x)$ of horizontal points (2)- $x_{\text {final, } \operatorname{cam}}(y)$. (e) Creation of a new horizontally corrected image, with horizontal length given by (3) (Fig. 5(c)), where the position in $x$ of each image point relative to the distorted image is given by (4)- $x_{c}$, $\operatorname{cam}(x, y)$. The $y$ coordinate of each point remains the same:

$x_{\text {initial, } \operatorname{cam}(y)=f(y)}$

$x_{\text {final, } \operatorname{cam}(y)}=f(y)$

$I_{\text {Widthc }}=W_{\text {max }}$ rectangle $N_{\text {rectangles }}$

$$
\begin{aligned}
& x_{\text {c,cam }}(x, y)=\frac{x-x_{\text {initial,cam }}(y)}{I_{\text {Widthc }} /\left(x_{\text {final,cam }}(y)-x_{\text {initial,cam }}(y)\right)} \\
& 0 \leqslant x<I_{\text {Width }} ; \quad 0 \leqslant y<I_{\text {Height }} ; 1 \leqslant \text { cam } \leqslant 2
\end{aligned}
$$

where cam is the camera number; $W_{\max }$ rectangle the dimension in pixels of the width of the widest image rectangle - this value must be equal for both images; $N_{\text {rectangles }}$ the number of rectangles between two points, the horizontal zero of the image, and the horizontal end limit - this value must be the same for both images; and $I_{\text {Widthc }}$ the image width after image horizontal correction-this value must be the same for both images.

\subsection{Vertical image correction}

The vertical image correction (Fig. 5(b)) for each camera is divided in the following steps (the points acquired from each image will have to correspond to the vertical limits of the selected vision field):

(a) Acquisition of a set of horizontal points from the image, which will be the vertical zeros of the image after vertical image correction. 
(b) Polynomial determination that translates the relation between the position $(x)$ of the horizontal points and the

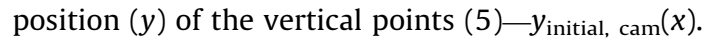

(c) Acquisition of a set of horizontal points from the image, which will be the vertical end limits of the image after vertical image correction.

(d) Polynomial determination that translates the relation between the position $(x)$ of horizontal points and the position $(y)$ of vertical points $(6)-y_{\text {final, } \operatorname{cam}}(x)$.

(e) Creation of a new vertically corrected image, with horizontal length given by (7) (Fig. 5(d)), where the position in $y$ of each image point relative to the horizontally corrected image, is given by $(8)-y_{c}, \operatorname{cam}(x, y)$. The $x$ coordinate of each point remains the same:

$$
\begin{aligned}
& y_{\text {initial, cam }}(x)=f(x) \\
& y_{\text {final, cam }}(x)=f(x) \\
& I_{\text {Heightc }}=H_{\text {max }} \text { rectangle } N_{\text {rectangles }} \\
& y_{\text {c, cam }}(x, y)=\frac{y-y_{\text {initial,cam }}(x)}{I_{\text {Heightc }} /\left(y_{\text {final,cam }}(x)-y_{\text {initial,cam }}(x)\right)} \\
& 0 \leqslant x<I_{\text {Widthc }} ; 0 \leqslant y<I_{\text {Height }} ; 1 \leqslant \operatorname{cam} \leqslant 2
\end{aligned}
$$

where $H_{\text {max rectangle }}$ is the dimension in pixels of the height of the highest image rectangle - this value must be equal for both images; $N_{\text {rectangles }}$ the number of rectangles between two points, the vertical zero of the image, and the vertical end limit-this value must be the same for both images; and $I_{\text {Heightc }}$ the image height after vertical image correction-this value must be the same for both images.

\subsection{Real-world relationships}

The relationship between the corrected image points and the points of $O_{\text {Laser }}$ plane (Fig. 5(d)), in $x$ coordinate, is obtained by (9), where $k$ is a constant. This constant, $k$, is determined by the division of the vertical length of one rectangle pattern of the calibrated image and the vertical length of one rectangle of the physical pattern ( $10 \mathrm{~mm}$ in the pattern used).

$x_{\text {Real }}=k \times x, \quad 0 \leqslant x<x_{\text {Widthc }}$

In $y$ coordinate, the relationship is obtained by (10), where $f(y)$ is a polynomial. This polynomial is defined by the acquisition of a set of vertical points in the corrected image. These points must represent a point on each horizontal edge of the rectangle in the corrected image, once the real distance between edges is known (through the physical pattern)

$y_{\text {Real }}(y)=f(y), \quad 0 \leqslant y<Y_{\text {Heightc }}$

The selection of points in each step of the calibration procedure was done manually. Several methods for point fitting were tested. The best method, resulting in a smaller mean squared error, was a $3^{\circ}$ polynomial approximation. For the determination of the polynomial, in both calibration steps, the least-squares method was used.

\section{Laser line detection}

The accuracy of a 3D measurement, which uses laser scanners, is significantly affected by the detection of the laser line. The energy pattern of such a line corresponds to a Gaussian profile. The laser line detection method presented here is executed in two steps.
The first step, applied to both images, is carried out before the calibration process. This step is characterized by the first vertical derivative image calculation. Therefore, each image column is derived and the laser is detected (Fig. 6), using three validating conditions:

(a) to search in the derived signal (column) for positive to negative transitions and the associated peaks;

(b) to verify if the distance between peaks is greater than $\Delta_{\text {Laser }}$ Height-maximum number of vertical laser pixels;

(c) to calculate the amplitude between peaks and to verify if the amplitude is greater than $\Delta_{\text {Laser_Intensity-laser minimum }}$ intensity relative to the surface on which it occurs.

If the three conditions are positive, the vertical pixels of the analysed column, between transitions, are placed in white and the remaining in black. In the event of detecting more than one zone with valid parameters in the same column, the one with greater $\Delta_{\text {Laser_Intensity }}$ is chosen. The value of the parameter $\Delta_{\text {Laser_Height }}$ is attributed according to the vertical resolution of the camera, the laser power and the distance between camera and laser. The value of the parameter $\Delta_{\text {Laser_Intensity }}$ is defined by analysing the intensity of the laser line over the material on which the measurements will be taken.

After the laser line detection in both the images, Sections 3.1 and 3.2 of the calibration procedure are executed. Then, a logic OR operation is carried out between the two calibrated images (Fig. 7).

The second step of the laser line detection consists of the detection of the laser line centre, which corresponds to the average value between the maximum and the minimum values. This step is carried out after Section 3.2 and before Section 3.3 of the calibration procedure. Then, the laser line centre is detected from the resultant image of the logical operation (Fig. 8). This image is then ready for execution in Section 3.3, the final step of the calibration procedure.

\section{Results and discussion}

To demonstrate the effectiveness of the calibration procedure, two different analyses were made. The first analysis carried out by the system developed had, as main incidence, the extremities of the system's vision field. As was previously verified, it was at the extremities of the images that the radial distortion caused by lens and the distortion caused by the cameras' orientation to the laser line projection plane were more perceptible. Therefore, two

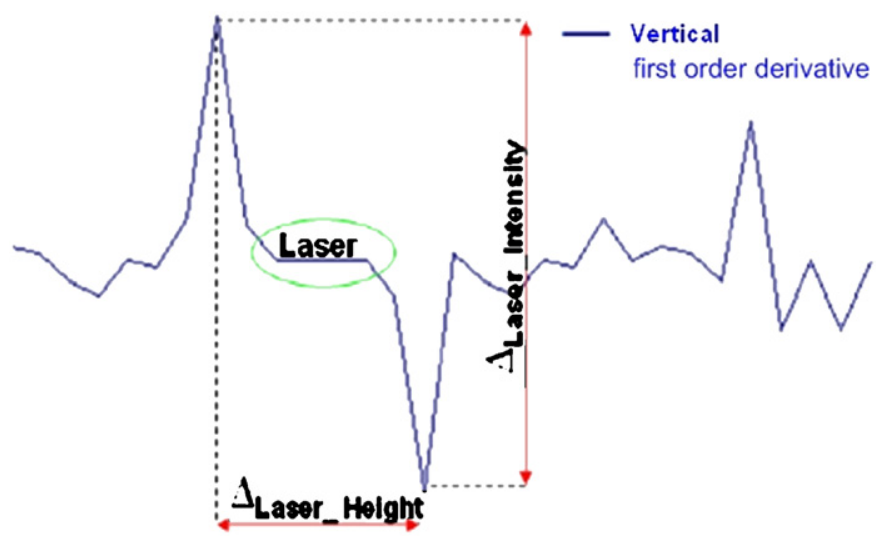

Fig. 6. Illustration of image vertical first-order derivate and laser line detection. 


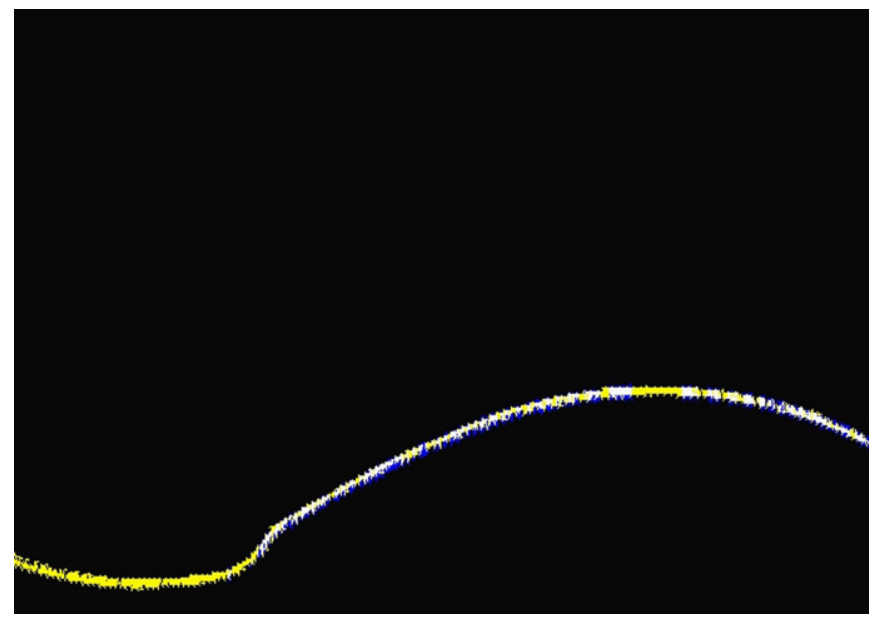

Fig. 7. Laser line detection result, by both cameras, after execution of the horizontal and vertical images calibration and logical OR: yellow-camera 1 image, blue-camera 2 image, white-camera 1 image and camera 2 image intersections.

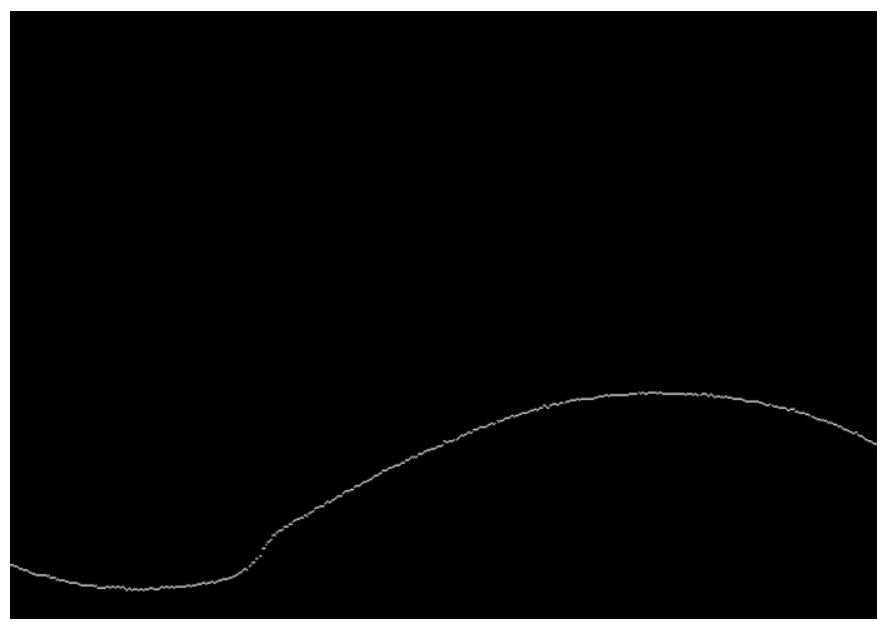

Fig. 8. Image resulting from the logic operation.

calibrated objects, with different types of geometry, were used for testing measurements in this system. The selected objects present the following geometric and dimensional characteristics:

Calibre 1-Stepper calibre (Fig. 9(a))-with horizontal and vertical steps of approximately $10 \mathrm{~mm}$.

Calibre 2-Variable radius cylinder calibre (Fig. 9(b)) - with two control radius points;

A high precision CMM (contact measure machine) was used to obtain the height and length values of each step of the calibre 1 and the two radius values of the calibre 2 (Mitutoyo, Class 0 , Precision $0.1 \mu \mathrm{m}$ ).

A set of measurements of the calibre 1 (height and length) was performed using the system developed. The measurements were made with the calibre placed in different points of the image, with special incidence on the image borders, because those are the regions of greatest distortion. Fig. 10(a) and (b) shows the measurements made by the developed system, respectively, of $X$-axis (horizontal) and $Y$-axis (vertical). The statistical results are presented in Table 1.

To obtain the value of the radius of the circles of calibre 2 , three measurements were made in different points of each circle. The
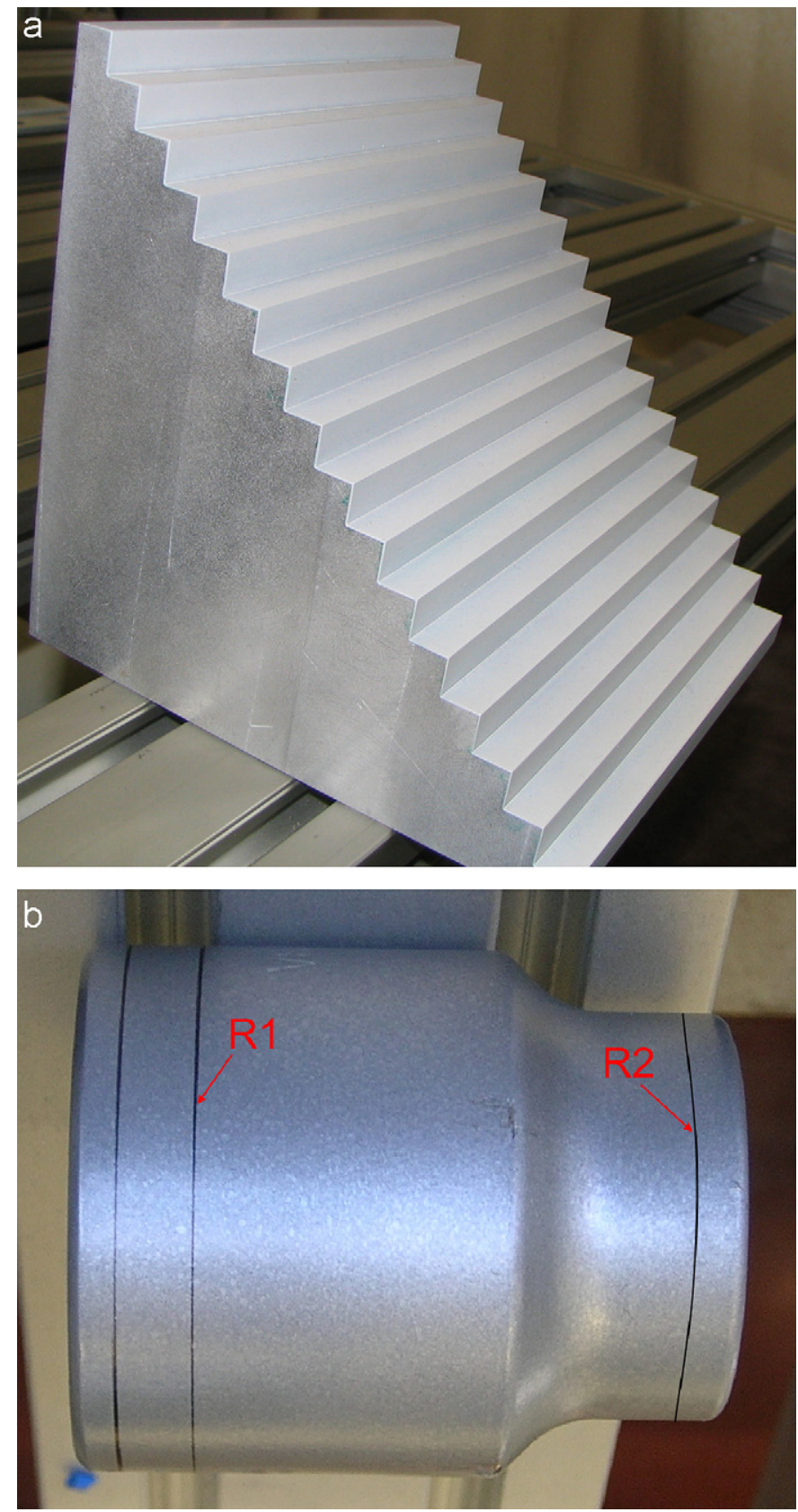

Fig. 9. Calibres used in the calibration procedure: (a) stepper calibre, (b) cylindrical with variable radius calibre.

measurements of calibre 2 were made in different points of the image, such as the measurements of calibre 1, with special incidence in the image extremities. Table 2 shows the measurements results made by the system for, respectively, radius $1(R 1)$ and radius $2(R 2)$. The statistical results are shown in Table 3.

From the results analysis, it is possible to say that:

- The system presents a $0.25 \mathrm{~mm} /$ pixel horizontal resolution and a $0.33 \mathrm{~mm} /$ pixel vertical resolution, in a vision field of $170 \times 160 \mathrm{~mm}^{2}$.

- The maximum error is smaller than 2 pixels, which expresses a system reading error smaller than $0.5 \mathrm{~mm}$, in both orientations (vertical and horizontal). The system error is subject to improvement by using higher resolution cameras or lenses 
a
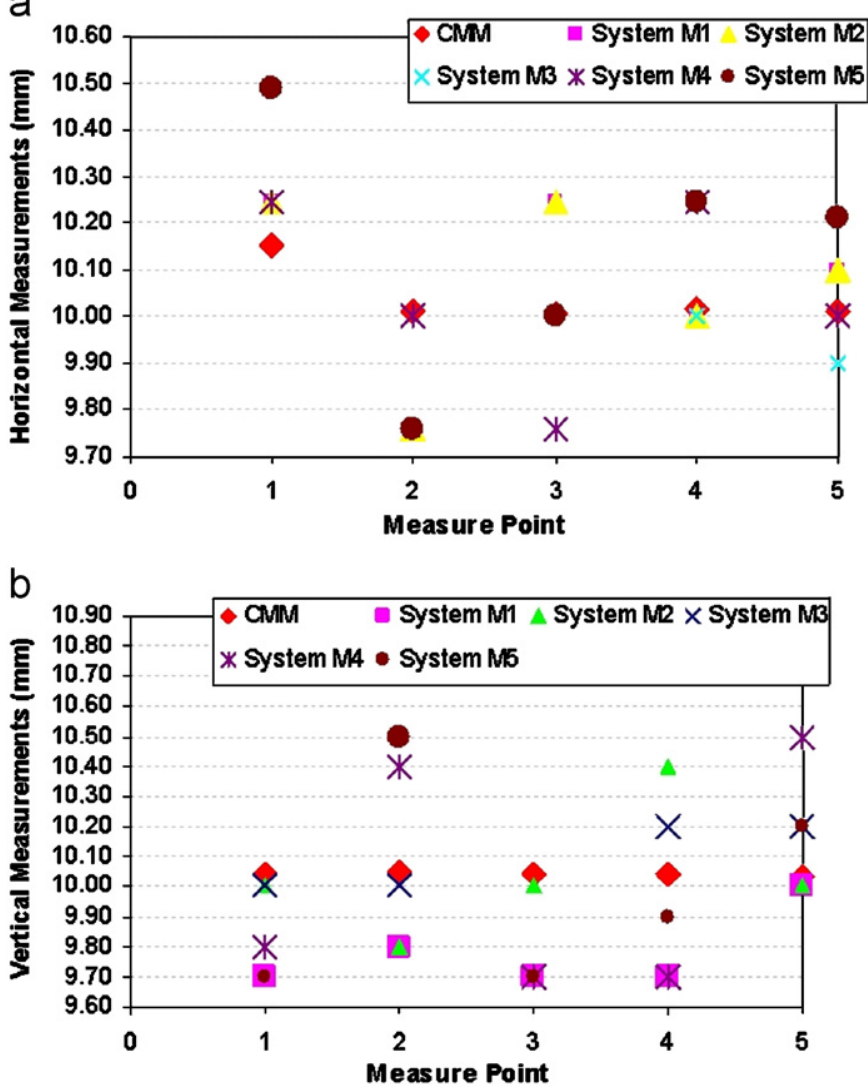

Fig. 10. Calibration results: (a) chart with five calibre 1 horizontal step measurements ( $X$-axis) on five distinguished image points (system M1-M5), (b) chart with five calibre1 vertical step measurements ( $Y$-axis) on five determined image points (system M1-M5).

Table 1

Calibre 1 -horizontal and vertical error measurements results

\begin{tabular}{lrlll}
\hline Error measure type & Mean & Absolute min & Absolute max & Std \\
\hline Horizontal (unit: mm) & 0.05 & 0.25 & 0.34 & 0.19 \\
Vertical (unit: mm) & -0.03 & 0.34 & 0.36 & 0.23 \\
\hline
\end{tabular}

Table 2

Calibre 2-radius measurements in five different image regions

\begin{tabular}{lcc}
\hline Measurement system & Radius $R 1$ & Radius $R 2$ \\
\hline CMM & 31.02 & 24.05 \\
Developed system & & \\
M1 & 31.17 & 23.83 \\
M2 & 30.76 & 24.06 \\
M3 & 30.99 & 23.84 \\
M4 & 30.75 & 24.23 \\
M5 & 31.23 & 23.91 \\
\hline
\end{tabular}

Table 3

Calibre 2-radius error measurements results

\begin{tabular}{lllll}
\hline Calibre radius & Mean & Absolute min & Absolute max & Std \\
\hline 1 (unit: $\mathrm{mm}$ ) & 0.03 & 0.22 & 0.27 & 0.21 \\
2 (unit: $\mathrm{mm}$ ) & 0.04 & 0.18 & 0.21 & 0.17
\end{tabular}

that reduce the vision field. Therefore, increasing the system resolution results in a less significant pixel error.

- The standard derivation is approximately the same in both calibre measurements.

- The mean error is practically zero in both measurements, suggesting that the error fluctuates above and below the real measure value.

- The mean error is smaller than the system resolution.

- Easy laser line detection on surfaces with high level of laser line absorption and complex geometry.

The results achieved validate the use of this calibration procedure in this system.

The second analysis of the system developed was executed on a road surface texture. This analysis is intended to demonstrate the possibility of a practical application of this system in the dimensional inspection of this surface type.

For this purpose, a comparative study of a complex line profile was made using a CMM (also used for calibre measurement) and the system developed (Fig. 11). The sample distance was of $1 \mathrm{~mm}$. The acquired sample presents a complex profile. This reality frustrates the profile acquisition by the CMM in some narrow concavities, as it is the case of the intervals $\{23,28\}$ and $\{30,40\}$. Table 4 shows the statistical comparison between the two acquisition systems. Looking at the results it is possible to emphasize that:

- The mean value of the CMM system is greater $0.36 \mathrm{~mm}$ than the mean value of the developed system. This fact could be influenced by the scanner system error and the CMM difficulty to reach narrow concavities.

- The maximal and minimum vertical values measured in several acquisition points present similar results in both systems.

- The maximal acquisition point has almost the same value for both systems.

- The standard derivation is higher in the system developed than in the CMM system; this is due to the same factors that affect the measured mean value.

\section{Conclusion}

This paper presents a new stereo vision calibration procedure based on the laser line projection plane. The calibration procedure, while using only laser-coplanar points, is oriented towards laser line detection, allows the matching of two images on the laser plane without the use of a rigid motion equation and correcting two kinds of distortion: radial distortion and distortion provoked by the orientation of the camera relative to the laser line projection plane.

The system implemented is composed of two CCD FIREWIRE cameras, with a resolution of $640 \times 480$, using $8 \mathrm{~mm}$ focal length lenses and a $635 \mathrm{~nm}$ wavelength, $1 \mathrm{~mW}$ power laser line, presenting a reading error smaller than $0.5 \mathrm{~mm}$. The system also allows easy laser line detection in surfaces with high level of laser line absorption and complex geometry.

These characteristics allow its usage for road surface textures acquisition, for macro and mega textures analysis, as was demonstrated by the second analysis described in Section 5. It is also possible to use it for other applications with this kind of requirements, such as sand movement acquisition-a laboratory simulation of the sand movements in rivers near bridge supports. 


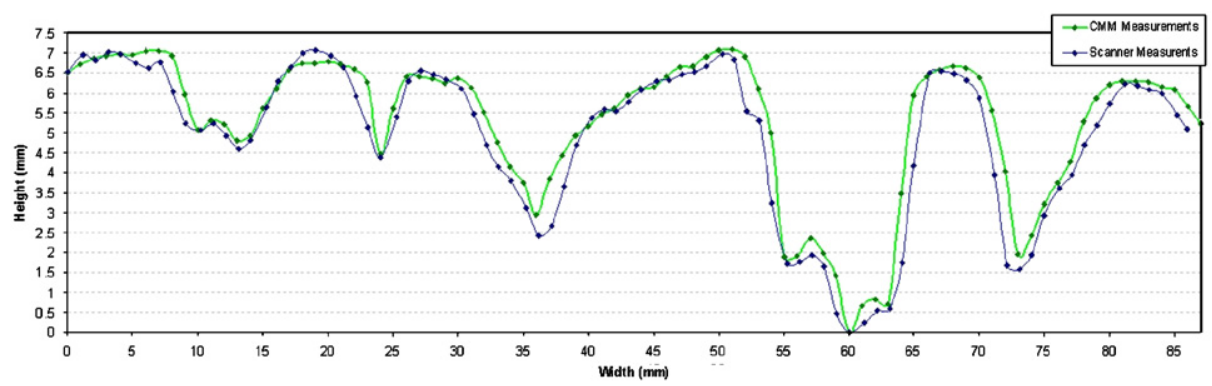

Fig. 11. This graph establishes a comparison between a line points acquisition carried out by the developed scanner system and the CMM over road profile surface.

Table 4

Complex line profile acquisition: CMM versus developed system

\begin{tabular}{lcccc}
\hline Measurement system & Mean & Min & Max & Std \\
\hline CMM (unit: mm) & 5.29 & 0.00 & 7.09 & 1.76 \\
Developed system (unit: $\mathrm{mm}$ ) & 4.93 & 0.01 & 7.08 & 1.92 \\
\hline
\end{tabular}

\section{Acknowledgement}

This work has been supported by "Fundação para a Ciência e a Tecnologia" (Portugal) through the Ph.D. grant, reference SFRH/ $\mathrm{BD} / 18155 / 2004$.

\section{References}

[1] Yau Hong-Tzong, Chen Chun-Yan, Wilhelm Robert G. Registration and integration of multiple laser scanned data for reverse engineering of complex 3D models. Int J Prod. Res 2000;38(2):269-85.
[2] Aguilar JJ, Torres F, Lope MA. Stereo vision for 3D measurement: accuracy analysis, calibration and industrial applications. Measurement 1996;18(4): 193-200.

[3] Lenz Reimar K, Tsay Roger Y. Techniques for calibration of scale factor and image center for high accuracy 3-D machine vision metrology. IEEE Trans Pattern Anal Mach Intell 1988;10(5).

[4] Tsai Roger Y. A Versatile camera calibration technique for high accuracy 3D machine vision metrology using off-the-shelf TV cameras and lenses. IBM research report RC 51342, May 8, 1985.

[5] Heikkila J, Silve' n O. A four-step camera calibration procedure with implicit image correction. In: Proceedings of CVPR ‘97, IEEE 1997 p. 1106-1112.

[6] Zhang Z. A flexible new technique for camera calibration. IEEE Trans Pattern Anal Mach Intell 2000;22(11):1330-4.

[7] Sturm P, Maybank S. On plane-based camera calibration: a general algorithm, singularities, applications. Proc IEEE CVPR 1999:432-7.

[8] França João G, Gazziro, Mário A, Ide Alessandro N, Saito, José H. A 3D scanning system based on laser triangulation and variable field of view. In: Conference on image processing, IEEE, 2005

[9] Devernay F, Faugeras O. Computing differential properties of 3-D shapes from stereoscopic images without 3-D models. In: CVPR'94, IEEE 1994 p. 208-213.

[10] Barone, Sandro, Bruno. Stereo vision and laser stripers for three-dimensional surface measurements. In: Andrea, XVI congreso international de ingeniería Gráfica, 2004 